\title{
Leveraging Single Atom Dynamics to Measure the Electron-Beam-Induced Force and Atomic Potentials
}

Ondrej Dyck, ${ }^{1,2}$ Feng Bao, ${ }^{3}$ Maxim Ziatdinov, ${ }^{1,2}$ Ali Yousefzadi Nobakht, ${ }^{4}$ Seungha Shin, ${ }^{4}$ Kody Law, ${ }^{5,6}$ Artem Maksov, ${ }^{1,2,7}$ Bobby G. Sumpter, ${ }^{1,2}$ Richard Archibald, ${ }^{1,5}$ Stephen Jesse, ${ }^{1,2}$ and Sergei V. Kalinin ${ }^{1,2}$

1. The Institute for Functional Imaging of Materials, Oak Ridge National Laboratory, Oak Ridge, TN, USA

2. The Center for Nanophase Materials Sciences, Oak Ridge National Laboratory, Oak Ridge, TN, USA

3. Department of Mathematics, The University of Tennessee at Chattanooga, Chattanooga, TN, USA

4. Department of Mechanical, Aerospace, and Biomedical Engineering, the University of Tennessee, Knoxville, TN, USA

5. Computer Science and Mathematics Division, Oak Ridge National Laboratory, Oak Ridge, TN, USA

6. School of Mathematics, University of Manchester, Manchester, UK

7. Bredesen Center for Interdisciplinary Research, University of Tennessee, Knoxville, TN, USA

In the last decade many examples of electron-beam-mediated chemical transitions have emerged, driving forward the idea that the transmission electron microscope may be utilized as an atomic scale fabrication platform.[1-3] However, precise knowledge of the undergirding beam-sample interactions at the atomic scale is still lacking. In order to harness the variety of reaction pathways observed under electron beam irradiation, account must be taken of not only the atomic potentials within the sample but also energy deposited by the beam. Here, we show that a single dopant Si atom in a graphene lattice can be used as an atomic scale force sensor, uncovering information on the random force exerted by the electron beam on chemically-relevant time scale. Using a stochastic reconstruction of molecular dynamic simulations, we recover the potential energy landscape of the atom, and leverage this information to determine the experimental beam-induce force. We further demonstrate that a moving atom attached to a graphene step edge can be used to map potential energy along the edge. These studies open the pathway for quantitative studies of beam-induced atomic dynamics and predictive atom-by-atom fabrication.

Figure 1 a) shows a high angle annular dark field (HAADF) image of a 4-fold coordinated Si substitutional dopant atom in a graphene lattice (artificially colored). The streaks in the image are due to atomic motion within the potential well brought on by energy transfer from the electron beam. Modelling the atomic intensity as a 2D gaussian, we are able to fit a 1D gaussian to each line of the image intensity, b), and retrieve a $2 \mathrm{D}$ map of the atomic positions observed during the image acquisition, $\mathrm{c}$ ). Molecular dynamics simulations of this structure under a periodic, stochasticly applied force, d), allow the reconstruction of a dynamic potential (i.e. taking into account phonon vibrations and the influx of beam energy), e) and f). Knowledge of the dynamic potential in comparison to the experimentally determined atomic positions enables determination of the force applied to the atom by the beam.

With this knowledge we may then apply the electron beam influence to a more complicated system. Here, we examine a circular step edge where a bilayer sheet of graphene has a hole through one layer and two foreign atoms are attached to this edge. Analysis of the video of the atom dynamics brought on by the electron beam is shown in $\mathrm{g}$ ) and $\mathrm{h}$ ). $\mathrm{g}$ ) is the sum of all frames of the video where we can observe the most stationary positions as bright spots. A map of each distinct position observed in the video is shown in $\mathrm{h}$ ). i) shows the telegraph-style atomic motion through time. With this detail we can combine the 
knowledge of the force from the electron beam with the observed atomic motion to determine the 1D potential along the step edge for each atom.

\section{References:}

[1] Jiang, N. et al, MRS Bulletin. 42 (2017), p. 653-659.

[2] Kalinin, S.V. and S.J. Pennycook, MRS Bulletin. 42 (2017), p. 637-643.

[3] Zhao, X. et al, MRS Bulletin. 42 (2017), p. 667-676.

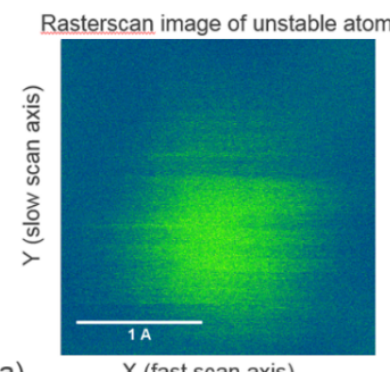

a)

d)

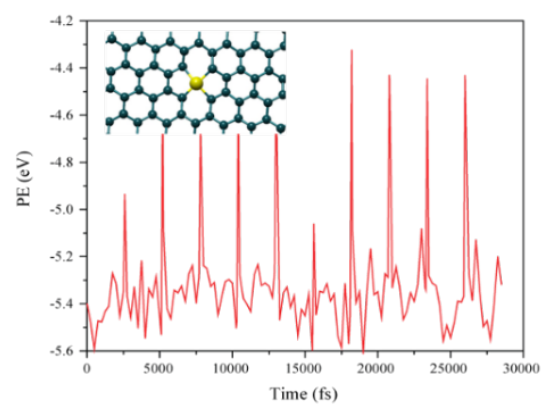

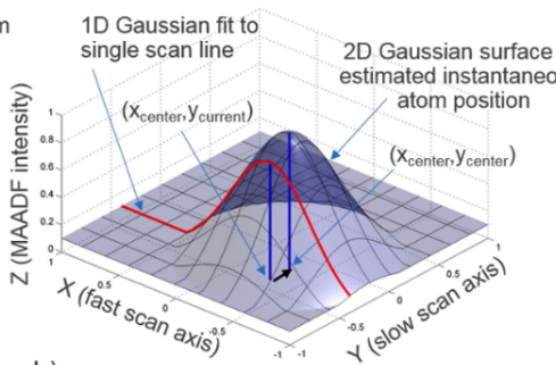

b)

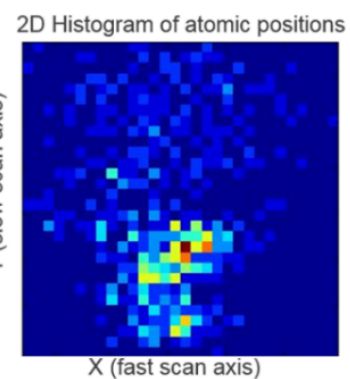

c)
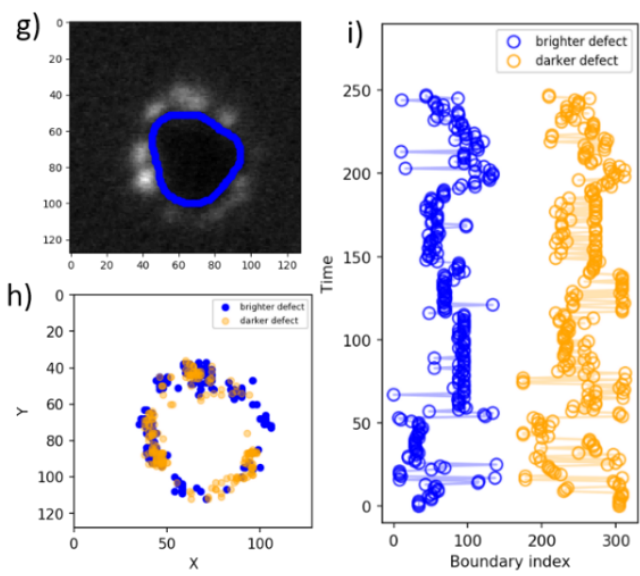

Figure 1 Illustration of applying the observation of atomic motion in combination with molecular dynamics to retrieve the atomic potential and force imparted by the electron beam to the atom. a) is a false-color HAADF image of a single 4-fold coordinated Si dopant in a graphene lattice. b) illustrates the fitting procedure where the atomic position for each scan line in a) is determined by gaussian fitting. The obtained position map is shown in c). d) shows the atomic model (inset) used for the molecular dynamics simulation and the energy of the atom through time under the application of a periodic stochastic force. The positional information is summarized in e), and the dynamic potential obtained is shown as a heat map in f) from which an estimate for the applied atomic force from the experimental data can be obtained. g) shows the sum of a video of two atoms moving along a step edge in bilayer graphene where the stable positions can be seen as bright spots. The single layer portion is circled in blue. $h$ ) illustrates each detected atomic position and i) shows the position through time. Knowledge of the beam position and applied force allows the $1 \mathrm{D}$ edge potential to be determined directly from experiment. 